\title{
Degraded Multicasting with Network Coding over the Combination Network
}

\author{
February 2, 2012
}

\begin{abstract}
In this paper, we give a characterization of the rate region for the degraded two message set problem, applied to a combination network with erasure channels. We also provide an algorithm that uses topological information in order to deliver the two messages to the receivers, and we show that our algorithm is optimal, in the sense that it achieves any rate pair in the region. We compare our algorithm analytically with a naive approach oblivious to the network structure, and we give an insight on what benefits should be expected for different classes of networks.
\end{abstract}

\section{Introduction}

Content delivery, i.e., multicasting, is an application where network coding promises to have impact, as significant benefits have been observed both theoretically as well as in practice. The case where all receivers require the exact same content is by now well understood; however, for the (perhaps more realistic) case, where different users require different subsets of the content, although there exist a number of proposed heuristic algorithms, there is in general no exact characterization of the optimal achievable rate region [1].

In this paper, we provide such a characterization for the degraded two-message set problem, where a source broadcasts two messages to a set of receivers over a combination network with erasure channels. Degraded broadcasting refers to that the "weaker" receivers receive a subset of the information that the "stronger" users collect. That is, the weaker users require a message $W_{1}$, transmitted at a rate $R_{1}$, while the stronger users require not only $W_{1}$, but also a second message $W_{2}$, transmitted at a rate $R_{2}$.

Degraded broadcasting is motivated by various scenarios, such as video streaming applications, or broadcasting in the presence of fading. In the first case, users 
are heterogeneous and have different subscription levels, thus requiring a different resolution of the content [9]. In the second case, the receivers are not able to receive the whole content due to channel fading, that can be modeled as erasures at higher layers.

The problem we solve is a special case of a long-standing open question in multi-user information theory, of delivering a set of degraded messages over a general broadcast channel introduced in [2]. Although special cases have been addressed $[3,4,5]$, there is comparatively little understanding when there are more than two users. Recent progress on a particular case of this question has been made in [7]. Closer to our work is the one in [8] that examines two-message broadcasting over a linear deterministic channel; our work differs in that we specifically look at the combination network, incorporate erasures, and provide a simpler achievability scheme.

Our main contributions in this paper are:

- We provide an exact characterization of the rate region for the two-degraded message-set problem, over the combination network and with three receivers.

- We present a very simple achievability scheme, that assigns source messages (or their linear combinations) to the network edges in linear time. A main observation from our work is that, to achieve the optimal rates, we need to take into account topological information, namely, what subset of receivers observes each edge.

- We provide an analytical comparison with a naive approach that is oblivious to the topology, and highlight what are the network topologies where the optimal approach can offer benefits.

A side result of our work is that, to achieve the optimal performance, we only need to use very simple binary network coding at a subset of the network edges.

The paper is organized as follows. We formulate the problem in Section 2 and give the characterization of the rate region $\mathcal{R}_{G}^{\alpha}$ for a combination network $G$, in the presence of erasures of rate $\alpha$ in Section 3. In Section 4 we introduce an algorithm that uses topological information to achieve any rate pair $\left(R_{1}, R_{2}\right) \in \mathcal{R}_{G}^{\alpha}$. Section 5 shows an analytical comparison between our algorithm and a naive network coding approach, where the resources are allocated without taking into account topological information. We conclude with some final remarks and directions for future work in Section 6. For the rest of the paper, we use the terms "edge" and "resource" interchangeably. 


\section{Problem formulation}

The problem of interest is communication of a public message $W_{1}$ and a private message $W_{2}$ at rates $R_{1}$, and $R_{2}$ respectively, to a set of three receivers, $\mathcal{U}=\{1,2,3\}$. The transmission is performed over a combination network $G$, illustrated in Fig. 1, where each channel has an erasure probability $\alpha$ and each receiver $i$ has access to $r_{i}$ edges. Message $W_{1}$ is required at all destinations, while message $W_{2}$ is only required at one of them, say the third receiver. Under this scenario, we set out to characterize the rate region $\mathcal{R}_{G}^{\alpha}$ at which messages $W_{1}$ and $W_{2}$ can be reliably communicated to the three receivers.

In this paper, we let $\mathcal{E}$ denote the total set of the intermediate edges, and $\mathcal{E}_{i} \subseteq \mathcal{E}$ denotes the set of the edges visible only to receiver $i$. Similarly $\mathcal{E}_{i j} \subseteq \mathcal{E}$ contains the edges visible only to receivers $i$ and $j$ and $\mathcal{E}_{i j k}$ is the set of edges visible to all three of the receivers. With this notation, we have that: $\mathcal{E}=\mathcal{E}_{1} \cup \mathcal{E}_{2} \cup \mathcal{E}_{3} \cup \mathcal{E}_{12} \cup$ $\mathcal{E}_{13} \cup \mathcal{E}_{23} \cup \mathcal{E}_{123}$, where each edge $e \in \mathcal{E}$ is visible to at least one receiver and it belongs to exactly one of the defined subsets.

Finally, we assume the size of the field over which the coding operations are performed is large enough, such that the linear combinations sent over the outgoing edges are independent with high probability. Thus, the number of linear independent combinations received by each destination $i$ is equal to $r_{i}$, the mincut to each destination, and it is given by: $r_{i}=\left|\mathcal{E}_{i}\right|+\sum_{j \in \mathcal{U}, j \neq i}\left|\mathcal{E}_{i j}\right|+\left|\mathcal{E}_{123}\right|$. In particular,

$$
\begin{aligned}
& r_{1}=\left|\mathcal{E}_{1}\right|+\left|\mathcal{E}_{12}\right|+\left|\mathcal{E}_{13}\right|+\left|\mathcal{E}_{123}\right| \\
& r_{2}=\left|\mathcal{E}_{2}\right|+\left|\mathcal{E}_{12}\right|+\left|\mathcal{E}_{23}\right|+\left|\mathcal{E}_{123}\right| \\
& r_{3}=\left|\mathcal{E}_{3}\right|+\left|\mathcal{E}_{13}\right|+\left|\mathcal{E}_{23}\right|+\left|\mathcal{E}_{123}\right|
\end{aligned}
$$

We also denote with $r_{i j}$ the size of the union of the edges that two destinations $i$ and $j, i \neq j$, observe. The received signal at receiver $i$ is given by $\bar{y}_{i}=\left[y_{i, 1} \cdots y_{i, r_{i}}\right]^{t}$ where $y_{i, j}$ is the signal received on the $j^{\text {th }}$ incoming edge of destination $i$. By $\bar{Y}_{i}^{n}=\left[y_{i, 1}^{n} \cdots y_{i, r_{i}}^{n}\right]^{t}$ we denote the received signals at receiver $i$ during a block length $n$. Similarly, the signal passing through edges of $\mathcal{E}_{3}$ is denoted by $\bar{y}_{\mathcal{E}_{3}}=\left[y_{\mathcal{E}_{3}, 1} \cdots y_{\mathcal{E}_{3},\left|\mathcal{E}_{3}\right|}\right]^{t}$

\section{Main result}

In this section, we characterize the capacity region of the degraded two message set scenario over a combination network with three receivers. 


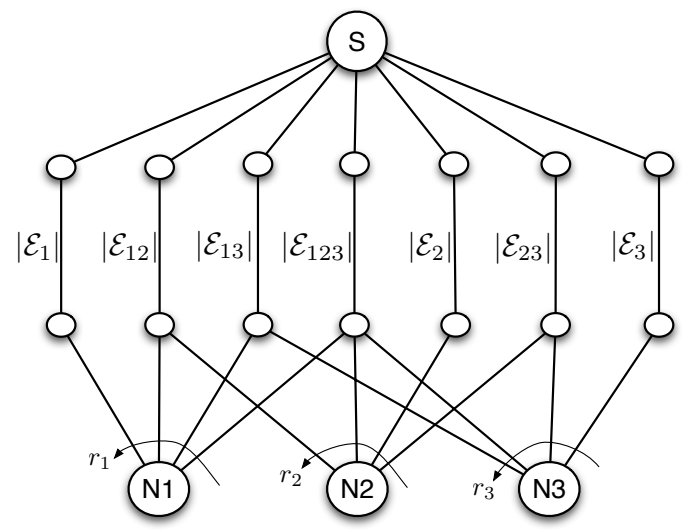

Figure 1: Combination network with one source and three receivers. For clarity, we represent every set $\mathcal{E}_{\{.\}}$using only one edge, and indicate set cardinality on the left side of that edge. Each receiver $i$ has access to $r_{i}$ edges.

Theorem 3.1 Any achievable rate pair $\left(R_{1}, R_{2}\right)$ in the degraded two message set scenario, applied over a combination network $G$ with channels of independent erasure probability $\alpha$ lies in the region $R_{G}^{\alpha}$ characterized by

$$
\begin{aligned}
R_{1} & \leq(1-\alpha) \min \left\{r_{i}\right\} \\
R_{1}+R_{2} & \leq(1-\alpha) r_{3} \\
2 R_{1}+R_{2} & \leq(1-\alpha)\left(r_{1}+r_{2}+\left|\mathcal{E}_{3}\right|\right)
\end{aligned}
$$

Theorem 3.2 Any rate pair $\left(R_{1}, R_{2}\right) \in \mathcal{R}_{G}^{\alpha}$ is achievable using the encoding scheme proposed by Algorithm 2.

We give the proof to Theorem 3.1 in this section and prove Theorem 3.2 in Section 4. 


\subsection{Proof of Theorem 3.1}

We prove here that $\mathcal{R}_{G}^{\alpha}$ characterizes an upper bound to $R_{1}$ and $R_{2}$.

$$
\begin{aligned}
n R_{1} & \leq H\left(W_{1}\right) \\
& \leq H\left(W_{1}\right)-H\left(W_{1} \mid \bar{Y}_{i}^{n}\right)+H\left(W_{1} \mid \bar{Y}_{i}^{n}\right) \\
& \stackrel{(a)}{\leq} H\left(W_{1}\right)-H\left(W_{1} \mid \bar{Y}_{i}^{n}\right)+n \epsilon_{i} \\
& \leq I\left(\bar{Y}_{i}^{n} ; W_{1}\right)+n \epsilon_{i} \\
& =H\left(\bar{Y}_{i}^{n}\right)-H\left(\bar{Y}_{i}^{n} \mid W_{1}\right)+n \epsilon_{i} \\
& \stackrel{(b)}{\leq} H\left(\bar{Y}_{i}^{n}\right)-H\left(\bar{Y}_{i}^{n} \mid X^{n}\right)+n \epsilon_{i} \\
& =I\left(\bar{Y}_{i}^{n} ; X^{n}\right)+n \epsilon_{i} \\
& \stackrel{(c)}{\leq} n r_{i}(1-\alpha)+n \epsilon_{i},
\end{aligned}
$$

where $\bar{Y}_{i}^{n}, i=1,2$, is the vector of received signals at receiver $i$. In the above chain of inequalities, $(a)$ follows for any $\epsilon_{i}>0$ by Fano's inequality, $(b)$ follows because $W_{1} \rightarrow X^{n} \rightarrow \bar{Y}_{i}^{n}$ forms a Markov chain, and (c) follows because the channels are discrete memoryless and parallel.

We furthermore get from (7) that $H\left(\bar{Y}_{i}^{n} \mid W_{1}\right) \leq H\left(\bar{Y}_{i}^{n}\right)-n R_{1}+n \epsilon_{i}$, and conclude that

$$
\begin{aligned}
I\left(\bar{Y}_{i}^{n} ; X^{n} \mid W_{1}\right) & \stackrel{(a)}{=} H\left(\bar{Y}_{i}^{n} \mid W_{1}\right)-H\left(\bar{Y}_{i}^{n} \mid X^{n}\right) \\
& \leq I\left(\bar{Y}_{i}^{n} ; X^{n}\right)-n R_{1}+n \epsilon_{i} . .
\end{aligned}
$$

In (a), we have used the fact that $W_{1} \rightarrow X^{n} \rightarrow \bar{Y}_{i}^{n}$ forms a Markov chain.

Similarly, for any $\epsilon^{\prime}>0$,

$$
\begin{aligned}
n\left(R_{1}+R_{2}\right) & \leq H\left(W_{1} W_{2}\right) \\
& \leq H\left(W_{1} W_{2}\right)-H\left(W_{1} W_{2} \mid \bar{Y}_{3}^{n}\right)+n \epsilon_{3} \\
& \leq I\left(\bar{Y}_{3}^{n} ; W_{1} W_{2}\right)+n \epsilon_{i} \\
& \leq I\left(\bar{Y}_{3}^{n} ; X^{n}\right)+n \epsilon_{i}+n \epsilon_{3} \\
& \leq n r_{3}(1-\alpha)+n \epsilon_{3} .
\end{aligned}
$$


Finally for any $\epsilon>0$,

$$
\begin{aligned}
n R_{2} & \leq H\left(W_{2} \mid W_{1}\right) \\
& \leq H\left(W_{2} \mid W_{1}\right)-H\left(W_{2} \mid \bar{Y}_{3}^{n} W_{1}\right)+n \epsilon \\
& =I\left(\bar{Y}_{3}^{n} ; W_{2} \mid W_{1}\right)+n \epsilon \\
& \stackrel{(a)}{\leq} I\left(\bar{Y}_{1}^{n} \bar{Y}_{2}^{n} \bar{Y}_{3}^{n} ; X^{n} \mid W_{1}\right)+n \epsilon \\
& \leq I\left(\bar{Y}_{1}^{n} ; X^{n} \mid W_{1}\right)+I\left(\bar{Y}_{2}^{n} ; X^{n} \mid \bar{Y}_{1}^{n}\right)+I\left(\bar{Y}_{3}^{n} ; X^{n} \mid \bar{Y}_{1}^{n} \bar{Y}_{2}^{n}\right)+n \epsilon \\
& \stackrel{(b)}{\leq} I\left(\bar{Y}_{1}^{n} ; X^{n}\right)-n R_{1}+n \epsilon_{1}+I\left(\bar{Y}_{2}^{n} ; X^{n}\right)-n R_{1}+n \epsilon_{2}+H\left(\bar{Y}_{3}^{n} \mid \bar{Y}_{1}^{n}, \bar{Y}_{2}^{n}\right)-H\left(\bar{Y}_{3}^{n} \mid X^{n}\right)+n \epsilon \\
& \leq I\left(\bar{Y}_{1}^{n} ; X^{n}\right)-n R_{1}+n \epsilon_{1}+I\left(\bar{Y}_{2}^{n} ; X^{n}\right)-n R_{1}+n \epsilon_{2}+H\left(\bar{Y}_{\mathcal{E}_{3}}^{n}\right)-H\left(\bar{Y}_{\mathcal{E}_{3}}^{n} \mid X^{n}\right)+n \epsilon \\
& \stackrel{(c)}{\leq} n(1-\alpha)\left(r_{1}+r_{2}+\left|\mathcal{E}_{3}\right|\right)-2 n R_{1}+n \delta,
\end{aligned}
$$

In the above chain of inequalities, $(a)$ follows by $W_{2} \rightarrow\left(W_{1}, X^{n}\right) \rightarrow \bar{Y}_{3}^{n}$ forming a Markov chain. To obtain (b), we first use the fact that $\bar{Y}_{1}^{n} \rightarrow\left(W_{1}, X^{n}\right) \rightarrow \bar{Y}_{2}^{n}$ forms a Markov chain and we obtain $I\left(\bar{Y}_{2}^{n} ; X^{n} \mid \bar{Y}_{1}^{n}\right) \leq I\left(\bar{Y}_{2}^{n} ; X^{n} \mid W_{1}\right)$. We then apply inequality (8) for $\epsilon_{i}=\epsilon^{\prime}=\epsilon, i=1,2$. Finally, $(c)$ follows from the fact that $I\left(\bar{Y}_{\mathcal{E}_{3}}^{n} ; X^{n}\right) \leq n(1-\alpha)\left|\mathcal{E}_{3}\right|$.

\subsection{Discussion}

From the inequalities which characterize $\mathcal{R}_{G}^{\alpha}$, (4) and (5) are straightforward, as they essentially express min-cut conditions, while the third inequality and its effect on the rate region is more interesting, and we thus discuss it in more detail in the following.

Assume for simplicity that $\alpha=0$, what intuitively the third inequality says is that if the $r_{1}$ edges to the first destination do not sufficiently overlap with the $r_{2}$ edges to the second destination, we may need to use twice the bottleneck edges in the combination network (hence the factor of 2) for $W_{1}$ to reach both these receivers. Then the rate $R_{2}$ we can send to the third receiver is limited by the "leftover" edges,

$$
R_{2} \leq\left(r_{1}-R_{1}\right)+\left(r_{2}-R_{1}\right)+\left|\mathcal{E}_{3}\right|,
$$

i.e., the edges that only the third receiver sees, and the edges remaining after duplicating message $W_{1}$ at rate $R_{1}$ to reach the first two receivers.

More formally, depending on the parameters of the topology, i.e., the number of edges in each set $\mathcal{E}_{\{.\}}$, the third inequality becomes active only for those topologies where the following situation occurs:

$$
\min \left\{r_{1}, r_{2}, r_{3}\right\}+r_{3}>r_{1}+r_{2}+\left|\mathcal{E}_{3}\right| .
$$




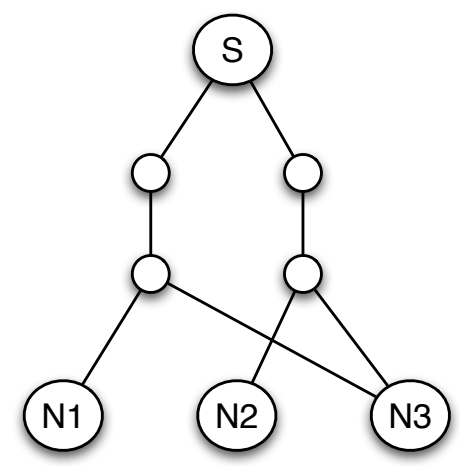

Figure 2: The third inequality is active

Note that if $r_{3}=\min \left\{r_{1}, r_{2}, r_{3}\right\}$, then the above relation does not hold, since $r_{i} \geq r_{3}, i \in\{1,2\}$. Therefore, $r_{3}$ does not affect the value of $\min \left\{r_{1}, r_{2}, r_{3}\right\}$ and we equivalently have the third inequality active when

$$
\min \left\{r_{1}, r_{2}\right\}+r_{3}>r_{1}+r_{2}+\left|\mathcal{E}_{3}\right| .
$$

Replacing the corresponding values of the ranks, we obtain that:

$$
\min \left\{\left|\mathcal{E}_{1}\right|+\left|\mathcal{E}_{13}\right|,\left|\mathcal{E}_{2}\right|+\left|\mathcal{E}_{23}\right|\right\}>\left|\mathcal{E}_{1}\right|+\left|\mathcal{E}_{2}\right|+\left|\mathcal{E}_{12}\right| .
$$

Fig. 2 is an example of such topological parameters. We give in the following an algorithm to verify for a desired combination network if the third inequality becomes active. We prove that Fig. 2 is the canonical combination network with the third inequality active; i.e., Algorithm 1 returns ACTIVE if and only if Fig. 2 is the combination network that remains after the edge eliminations up to that iteration.

The proof is in two steps. We first prove that the third inequality is active over a combination network $G$ if and only if it is active over the combination network $G^{\prime}$ which is obtained from $G$ after the edge elimination proposed by Algorithm 1 . We then argue that the third inequality is not active over all combination networks with $\left|\mathcal{E}_{13}\right|=0$ or $\left|\mathcal{E}_{23}\right|=0$. Finally, one should note that if $G$ is such that $\left|\mathcal{E}_{13}\right| \&\left|\mathcal{E}_{23}\right|>0$ and it cannot reduce thourgh Algorithm 1, then $\left|\mathcal{E}_{3}\right|=\left|\mathcal{E}_{123}\right|=$ $\left|\mathcal{E}_{12}\right|=\left|\mathcal{E}_{2}\right|=\left|\mathcal{E}_{1}\right|=0$.

Looking at (14), one notes that $\left|\mathcal{E}_{3}\right|$ and $\left|\mathcal{E}_{123}\right|$ do not play a role. This means that decreasing or increasing them does not affect the third inequality being active or not. Similarly, if $\left|\mathcal{E}_{12}\right|,\left|\mathcal{E}_{13}\right|$, and $\left|\mathcal{E}_{23}\right|$ increase or deacrese all three together, they will not affect (14). The same argument is valid for increasing or decreasing 


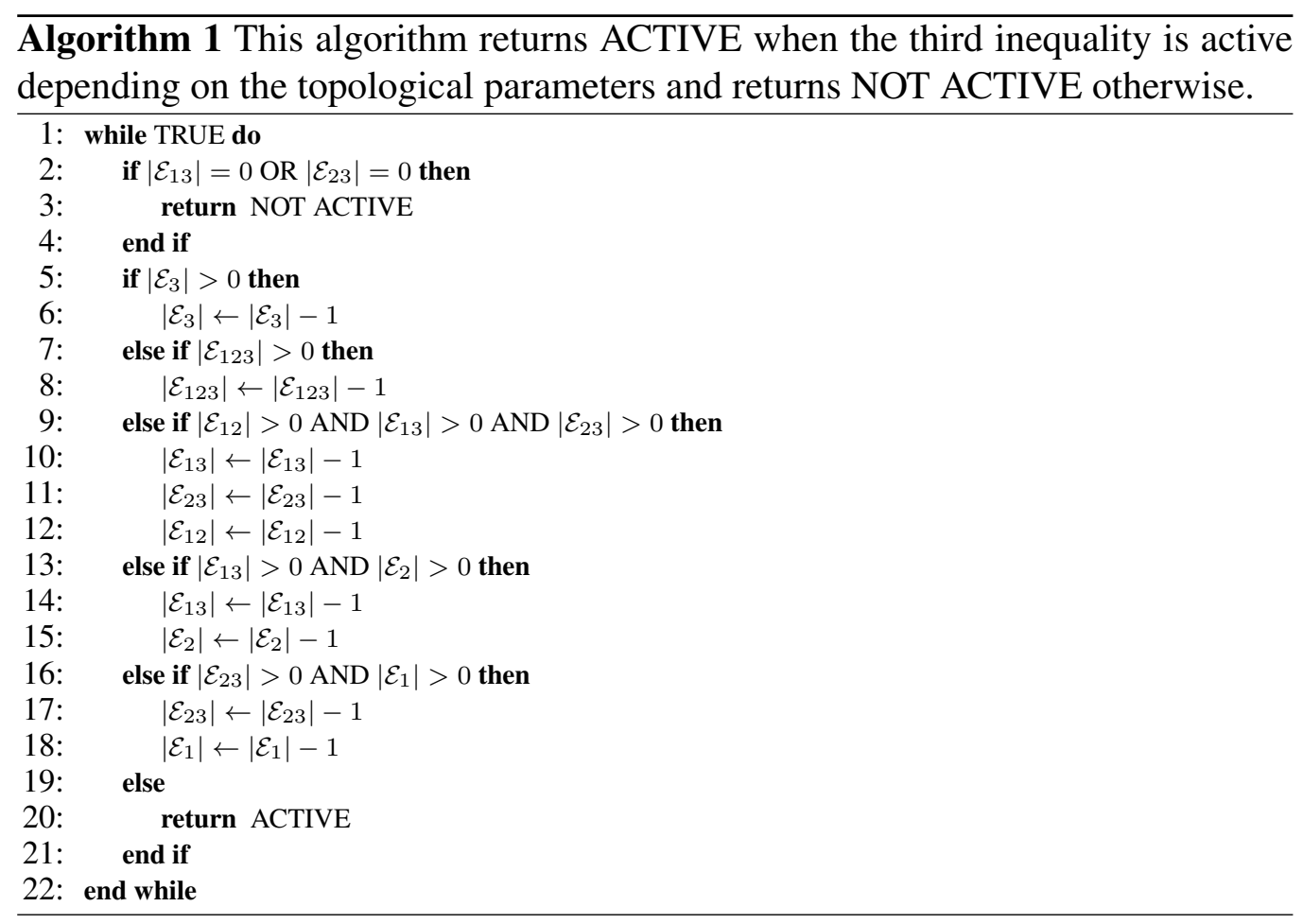

both of $\left|\mathcal{E}_{13}\right|$ and $\left|\mathcal{E}_{2}\right|$, or both of $\left|\mathcal{E}_{23}\right|$ and $\left|\mathcal{E}_{1}\right|$. So in each iteration of the while loop in Algorithm 1, a combination network is obtained which is equivalent (in terms of the tightness of third inequality) to the initial combination network $G$.

Furthermore, one readily verifies that if $\left|\mathcal{E}_{13}\right|=0$,

$$
\min \left\{\left|\mathcal{E}_{1}\right|+\left|\mathcal{E}_{13}\right|,\left|\mathcal{E}_{2}\right|+\left|\mathcal{E}_{23}\right|\right\} \leq\left|\mathcal{E}_{1}\right| \leq\left|\mathcal{E}_{1}\right|+\left|\mathcal{E}_{2}\right|+\left|\mathcal{E}_{12}\right| .
$$

Similarly, if $\left|\mathcal{E}_{23}\right|=0$,

$$
\min \left\{\left|\mathcal{E}_{1}\right|+\left|\mathcal{E}_{13}\right|,\left|\mathcal{E}_{2}\right|+\left|\mathcal{E}_{23}\right|\right\} \leq\left|\mathcal{E}_{2}\right| \leq\left|\mathcal{E}_{1}\right|+\left|\mathcal{E}_{2}\right|+\left|\mathcal{E}_{12}\right| .
$$

Finally, if $G$ is such that $\left|\mathcal{E}_{13}\right| \&\left|\mathcal{E}_{23}\right|>0$ and it cannot reduce thourgh Algorithm 1, then $\left|\mathcal{E}_{3}\right|=\left|\mathcal{E}_{123}\right|=\left|\mathcal{E}_{12}\right|=\left|\mathcal{E}_{2}\right|=\left|\mathcal{E}_{1}\right|=0$ which gives us the class of combination networks with the third inequality active ( Fig. 2).

\section{Algorithm description}

In this section we introduce an algorithm that uses topological information in order to achieve any desired rate pair $\left(R_{1}, R_{2}\right) \in \mathcal{R}_{G}^{\alpha}$. The algorithm uses the fact that each intermediate edge is essentially one available resource to the set of receivers that are connected to it and can carry linear combinations of $W_{1}$ and 
$W_{2}$. We show that we do not need to perform network coding among $W_{1}$ and $W_{2}$ in order to have an optimal algorithm (our Algorithm 2 is such an example). For the sake of simplicity we consider the case of no erasures in Section 4.1 and give the sketch of the proof for the case where each channel has an independent and uniform erasure probability of $\alpha$ in Section 4.2.

The idea of the algorithm is that the source puts linear combinations of symbols of $W_{1}$ or of $W_{2}$ on each of the edges so that it guarantees decodability of $W_{1}$ at all the receivers and decodability of $W_{2}$ at the third receiver. We are interested in assigning each resource to carry one of the two messages. We indicate this by coloring the intermediate edges with two colors, $t_{1}$ for $W_{1}$ and $t_{2}$ for $W_{2}$, where $t_{1} \neq t_{2}$ and both $t_{i}>0, i \in 1,2$. This edge assignment (edge coloring) is the output of our proposed Algorithm 2 for a given rate pair $\left(R_{1}, R_{2}\right) \in \mathcal{R}_{G}^{0}$. The algorithm makes use of two methods, which we explain briefly. Function FindEdge $(\mathcal{A})$ returns true if the set $\mathcal{A}$ contains at least an edge that has not been assigned for any message yet. Function $\operatorname{ColorEdge}\left(\mathcal{A}, t_{i}\right)$ marks an edge of the specified set $\mathcal{A}$ to carry message $W_{i}$.

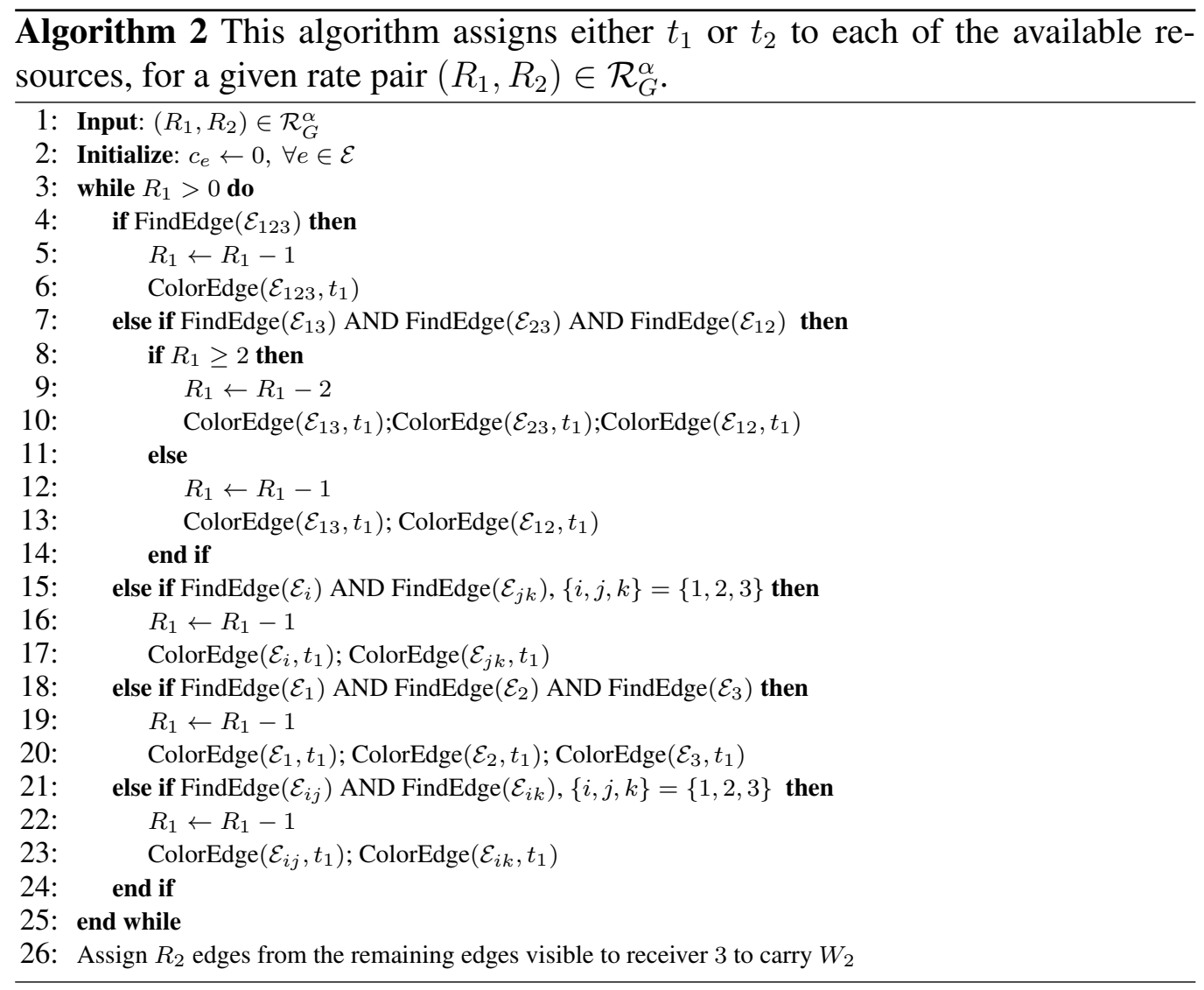

One should note that network coding is actually needed only for step 7 of $\mathrm{Al}$ - 
gorithm 2, when it assigns resources from the sets visible to all two receivers, $\mathcal{E}_{i j}$. By selecting an edge from each $\mathcal{E}_{i j}$, and sending a linear combination of $W_{1}$ on each of them, every destination receives a total rate of two. For the remaining situations, it is enough to route by conveniently selecting one edge from those sets that complement each other, for example sets $\mathcal{E}_{2}$ and $\mathcal{E}_{13}$ as long as the sets still contain edges that have not been assigned yet.

\subsection{Algorithm optimality - no erasures}

Lemma 4.1 Algorithm 2 stops after finite steps.

Proof We first prove that after each iteration (inside the while loop) $R_{1}$ is decreased by at least 1 . We then conclude that Algorithm 2 stops after at most $R_{1}$ iterations. In each iteration, $R_{1}$ is decreased if either of the "IF conditions" are satisfied. No "IF condition" is satisfied only when all $\left|\mathcal{E}_{123}\right|, \min \left\{\left|\mathcal{E}_{12}\right|,\left|\mathcal{E}_{13}\right|,\left|\mathcal{E}_{23}\right|\right\}$, $\min \left\{\left|\mathcal{E}_{i}\right|,\left|\mathcal{E}_{j, l}\right|\right\}, \min \left\{\left|\mathcal{E}_{1}\right|,\left|\mathcal{E}_{2}\right|,\left|\mathcal{E}_{3}\right|\right\}$ and $\min \left\{\left|\mathcal{E}_{i^{\prime}, j^{\prime}}\right|,\left|\mathcal{E}_{i^{\prime}, l^{\prime}}\right|\right\}$ are already assigned which ensures $R_{1}$ having been decreased by at least $r_{1}$ or $r_{2}$. But then since $\left(R_{1}, R_{2}\right) \in \mathcal{R}_{G}^{0}, R_{1}$ should satisfy $R_{1} \leq \min \left\{r_{1}, r_{2}\right\}$ which means that $R_{1} \leq 0$ in the studied iteration and this is a contradiction.

Lemma 4.2 Algorithm 2 is optimal

Proof We prove here that for any $\left(R_{1}, R_{2}\right) \in \mathcal{R}_{G}^{0}$, the assignment proposed by Algorithm 2 lets (i) all receivers get $R_{1}$ random linear combinations of $W_{1}$ and (ii) the third receiver further gets $R_{2}$ random linear combinations of $W_{2}$. The proof is by induction:

\section{Induction Base}

Let $R_{1}=0$. Algorithm 2 assigns $W_{2}$ to all the resources. Thus, receiver 3 gets $r_{3} \geq R_{1}+R_{2}$ random linear combinations of $W_{2}$ and (i) and (ii) both hold.

\section{Induction Hypothesis}

Let $R_{1} \leq r$ and assume that the assignment given by Algorithm 2 satifies (i) and (ii) for any $\left(R_{1}, R_{2}\right) \in \mathcal{R}_{G}^{0}$ and over all combination networks.

\section{Induction Step}

Assume $R_{1}=r+1$ and $\left(R_{1}, R_{2}\right) \in \mathcal{R}_{G}^{0}$. Run Algorithm 2 for one iteration to assign message $W_{1}$ on the edge(s) e that it finds, providing each receiver $k$, $k=1,2,3$ with $r_{k}^{\mathbf{e}} \geq 1$ linear combinations of $W_{1}$. We show that eliminating 
these edges leaves us with a combination network $G^{\prime}$ on which resources could be allocated to $\left(R_{1}-\min _{k}\left\{r_{k}^{\mathrm{e}}\right\}\right)$ rate of message $W_{1}$ and $R_{2}$ rate of message $W_{2}$. To this end, we show that $\left(R_{1}-\min _{k}\left\{r_{k}^{\mathrm{e}}\right\}, R_{2}\right) \in \mathcal{R}_{G^{\prime}}^{0}$, where $R_{1}-\min _{k}\left\{r_{k}^{\mathrm{e}}\right\} \leq r$ and $\mathcal{R}_{G^{\prime}}^{0}$ is the capacity region of the new combination network $G^{\prime}$. We then apply the induction hypothesis (which states that Algorithm 2 optimally gives the resource assignment on $G^{\prime}$ for all rate pairs $\left.\left(R_{1}^{\prime}, R_{2}^{\prime}\right) \in R_{G^{\prime}}^{0}, R_{1}^{\prime} \leq r\right)$ to conclude the optimality of Algorithm 2.

We take into account the following cases as suggested by Algorithm 2 and find the structure of $G^{\prime}$ which is formed after the edge elimination depending on the topology of the combination network.

1. $\left|\mathcal{E}_{123}\right|>0$. The edge to be marked in this case is an edge of $\mathcal{E}_{123}$. It is easy to see that $\min _{k}\left\{r_{k}^{\mathbf{e}}\right\}=1$ and the resulting $G^{\prime}$ has $r_{k}^{\prime}=r_{k}-1, k=1,2,3$, and $\left|\mathcal{E}_{3}^{\prime}\right|=\left|\mathcal{E}_{3}\right|$.

2. $\left|\mathcal{E}_{123}\right|=0$, and $\min \left\{\left|\mathcal{E}_{12}\right|, \mathcal{E}_{13}|,| \mathcal{E}_{23} \mid\right\}>0$. In this case, one edge from each $\mathcal{E}_{i j}$ is marked. We thus have $\min _{k}\left\{r_{k}^{\mathrm{e}}\right\}=2$ and $G^{\prime}$, depending on $R_{1}$, has either $r_{k}^{\prime}=r_{k}-2, k=1,2,3$, and $\left|\mathcal{E}_{3}^{\prime}\right|=\left|\mathcal{E}_{3}\right|$ (if $R_{1} \geq 1$ ) or $r_{1}^{\prime}=r_{1}-2$, $r_{2}^{\prime}=r_{2}-1, r_{3}^{\prime}=r_{3}-1$, and $\left|\mathcal{E}_{3}^{\prime}\right|=\left|\mathcal{E}_{3}\right|$ (if $R_{1}=1$ ).

3. $\left|\mathcal{E}_{123}\right|=\min \left\{\left|\mathcal{E}_{12}\right|,\left|\mathcal{E}_{13}\right|,\left|\mathcal{E}_{23}\right|\right\}=0$, and $\left|\mathcal{E}_{i}\right| \&\left|\mathcal{E}_{j, l}\right|>0$ for some $\{i, j, l\}=$ $\{1,2,3\}$. In this case, one edge from $\mathcal{E}_{i}$ and one edge from $\mathcal{E}_{j, l}$ is marked. So $\min _{k}\left\{r_{k}^{\mathrm{e}}\right\}=1$ and $G^{\prime}$ has the following topological parameters: $r_{k}^{\prime}=$ $r_{k}-1, k=1,2,3$, and either $\left|\mathcal{E}_{3}^{\prime}\right|=\left|\mathcal{E}_{3}\right|$ (if $i \neq 3$ ) or $\left|\mathcal{E}_{3}^{\prime}\right|=\left|\mathcal{E}_{3}\right|-1$ (if $i=3)$.

4. $\left|\mathcal{E}_{123}\right|=\min \left\{\left|\mathcal{E}_{12}\right|,\left|\mathcal{E}_{13}\right|,\left|\mathcal{E}_{23}\right|\right\}=\min \left\{\left|\mathcal{E}_{i}\right|,\left|\mathcal{E}_{j, l}\right|\right\}=0, \forall\{i, j, l\}=\{1,2,3\}$, and $\left|\mathcal{E}_{1}\right| \&\left|\mathcal{E}_{2}\right| \&\left|\mathcal{E}_{3}\right|>0$. In this case, one edge from each $\mathcal{E}_{i}$ is marked. Similarly, $\min _{k}\left\{r_{k}^{\mathrm{e}}\right\}=1$ and $G^{\prime}$ has $r_{k}^{\prime}=r_{k}-1, k=1,2,3$, and $\left|\mathcal{E}_{3}^{\prime}\right|=\left|\mathcal{E}_{3}\right|-1$.

5. $\left|\mathcal{E}_{123}\right|=\min \left\{\left|\mathcal{E}_{12}\right|,\left|\mathcal{E}_{13}\right|,\left|\mathcal{E}_{23}\right|\right\}=\min \left\{\left|\mathcal{E}_{i}\right|,\left|\mathcal{E}_{j, l}\right|\right\}=\min \left\{\left|\mathcal{E}_{1}\right|,\left|\mathcal{E}_{2}\right|,\left|\mathcal{E}_{3}\right|\right\}=$ $0, \forall\{i, j, l\}=\{1,2,3\}$, and $\left|\mathcal{E}_{i, j}\right| \&\left|\mathcal{E}_{i, l}\right|>0$ for some $\{i, j, l\}=\{1,2,3\}$. In this case, we have one edge from $\mathcal{E}_{i j}$ and one edge from $\mathcal{E}_{i l}$ marked. $\min _{k}\left\{r_{k}^{\mathrm{e}}\right\}=1$ and $G^{\prime}$ has $r_{i}^{\prime}=r_{i}-2, r_{j}^{\prime}=r_{j}-2, r_{l}^{\prime}=r_{l}-2$ and $\left|\mathcal{E}_{3}^{\prime}\right|=\left|\mathcal{E}_{3}\right|$.

In each of the mentioned cases we characterize $\mathcal{R}_{G^{\prime}}^{0}$ and show that $\left(R_{1}-\min _{k} r_{k}^{\mathrm{e}}, R_{2}\right) \in$ $\mathcal{R}_{G^{\prime}}^{0}$ for all $\left(R_{1}=r+1, R_{2}\right) \in \mathcal{R}_{G}^{0}$.

1. $\left|\mathcal{E}_{123}\right|>0 . \mathcal{R}_{G^{\prime}}^{0}$ is characterized by

$$
\begin{aligned}
R_{1}^{\prime} & \leq \min \left\{r_{1}, r_{2}, r_{3}\right\}-1 \\
R_{1}^{\prime}+R_{2}^{\prime} & \leq r_{3}-1 \\
2 R_{1}^{\prime}+R_{2}^{\prime} & \leq r_{1}-1+r_{2}-1+\left|\mathcal{E}_{3}\right| .
\end{aligned}
$$


Furthermore, $\min _{k} r_{k}^{\mathbf{e}}=1$ and so it's easy to verify that $\left(R_{1}-\min _{k} r_{k}^{\mathbf{e}}, R_{2}\right) \in$ $\mathcal{R}_{G^{\prime}}^{0}$ for all $\left(R_{1}=r+1, R_{2}\right) \in \mathcal{R}_{G}^{0}$.

2. $\left|\mathcal{E}_{123}\right|=0$, and $\min \left\{\left|\mathcal{E}_{12}\right|, \mathcal{E}_{13}|,| \mathcal{E}_{23} \mid\right\}>0$. This case is interestingly the only case where routing is not optimal and we consider two cases: $R_{1} \geq 2$ and $R_{1}=1$.

- $R_{1} \geq 2: \mathcal{R}_{G^{\prime}}^{0}$ is characterized by

$$
\begin{aligned}
R_{1}^{\prime} & \leq \min \left\{r_{1}, r_{2}, r_{3}\right\}-2, \\
R_{1}^{\prime}+R_{2}^{\prime} & \leq r_{3}-2, \\
2 R_{1}^{\prime}+R_{2}^{\prime} & \leq r_{1}-2+r_{2}-2+\left|\mathcal{E}_{3}\right| .
\end{aligned}
$$

Furthermore, $\min _{k} r_{k}^{\mathbf{e}}=2$. It is immediate to see that $\left(R_{1}-\min _{k} r_{k}^{\mathbf{e}}, R_{2}\right) \in$ $\mathcal{R}_{G^{\prime}}^{0}$ for all $\left(R_{1}=r+1>1, R_{2}\right) \in \mathcal{R}_{G}^{0}$.

- $R_{1}=1: \mathcal{R}_{G^{\prime}}^{0}$ is characterized by

$$
\begin{aligned}
R_{1}^{\prime} & \leq \min \left\{r_{1}-2, r_{2}-1\right\}, \\
R_{1}^{\prime}+R_{2}^{\prime} & \leq r_{3}-1, \\
2 R_{1}^{\prime}+R_{2}^{\prime} & \leq r_{1}-2+r_{2}-1+\left|\mathcal{E}_{3}\right| .
\end{aligned}
$$

Furthermore, $\min _{k} r_{k}^{\mathrm{e}}=1$. We prove by contradiction that for all $\left(R_{1}=1, R_{2}\right) \in \mathcal{R}_{G}^{0}$, we have $\left(R_{1}-\min _{k} r_{k}^{\mathbf{e}}=0, R_{2}\right) \in \mathcal{R}_{G^{\prime}}^{0}$. Assume that $\left(0, R_{2}\right) \notin \mathcal{R}_{G^{\prime}}^{0}$ for some $R_{2}$ which satisfies $\left(1, R_{2}\right) \in \mathcal{R}_{G}^{0}$. Then

$$
\min \left\{\begin{array}{l}
r_{3}-1 \\
r_{1}+r_{2}+\left|\mathcal{E}_{3}\right|-3
\end{array}\right\}<\min \left\{\begin{array}{l}
r_{3}-1 \\
r_{1}+r_{2}+\left|\mathcal{E}_{3}\right|-2
\end{array}\right\} .
$$

We show in the following that to have (26), we should have $r_{1}+r_{2}-$ $3+\left|\mathcal{E}_{3}\right|<r_{3}-1<r_{1}+r_{2}-2+\left|\mathcal{E}_{3}\right|$ which is a contradiction (for our assumed integer values): The right hand side can be simplified to $r_{3}-1$ and furthermore

$$
\begin{aligned}
r_{3}-1 \stackrel{(1)}{=} & \left|\mathcal{E}_{3}\right|+\left|\mathcal{E}_{13}\right|+\left|\mathcal{E}_{23}\right|-1 \\
\leq & \left|\mathcal{E}_{3}\right|+\left|\mathcal{E}_{13}\right|+\left|\mathcal{E}_{23}\right|+\left|\mathcal{E}_{1}\right|+ \\
& +\left|\mathcal{E}_{2}\right|+2\left(\left|\mathcal{E}_{12}\right|-1\right)-1 \\
& \stackrel{(2)}{=} \\
& r_{1}-1+r_{2}-3+\left|\mathcal{E}_{3}\right| \\
& <r_{1}-1+r_{2}-2+\left|\mathcal{E}_{3}\right|,
\end{aligned}
$$

where (1) and (2) are both by the assumption of $\left|\mathcal{E}_{123}\right|=0$. The left hand side is thus not equal to $r_{3}-1$, forcing $r_{1}-1+r_{2}-3+\left|\mathcal{E}_{3}\right|<$ $r_{3}-1<r_{1}-1+r_{2}-2+\left|\mathcal{E}_{3}\right|$ : contradiction. So $\left(R_{1}-\min _{k} r_{k}^{\mathrm{e}}=\right.$ $\left.0, R_{2}\right) \in \mathcal{R}_{G^{\prime}}^{0}$ for all $\left(R_{1}=1, R_{2}\right) \in \mathcal{R}_{G}^{0}$. 
3. $\left|\mathcal{E}_{123}\right|=\min \left\{\left|\mathcal{E}_{12}\right|,\left|\mathcal{E}_{13}\right|,\left|\mathcal{E}_{23}\right|\right\}=0$, and $\left|\mathcal{E}_{i}\right| \&\left|\mathcal{E}_{j, l}\right|>0$ for some $\{i, j, l\}=$ $\{1,2,3\}$. If $i \neq 3$, we have the same $\mathcal{R}_{G^{\prime}}^{0}, \min _{k} r_{k}^{\mathbf{e}}$, and thus exactly the same argument as when $\left|\mathcal{E}_{123}\right|>0$. If $i=3$, then from $\min \left\{\left|\mathcal{E}_{j 3}\right|,\left|\mathcal{E}_{l 3}\right|\right\}=0$ and $\left|\mathcal{E}_{12}\right|>0$, we find that

$$
\min \left\{\left|\mathcal{E}_{1}\right|+\left|\mathcal{E}_{13}\right|,\left|\mathcal{E}_{2}\right|+\left|\mathcal{E}_{23}\right|\right\}<\left|\mathcal{E}_{1}\right|+\left|\mathcal{E}_{2}\right|+\left|\mathcal{E}_{12}\right|
$$

which infact turned out to be the condition for the third inequality not being active. In all such cases, $\mathcal{R}_{G}^{0}$ is characterized by

$$
\begin{aligned}
R_{1}^{\prime} & \leq \min \left\{r_{1}, r_{2}\right\} \\
R_{1}^{\prime}+R_{2}^{\prime} & \leq r_{3}
\end{aligned}
$$

and as a result $\mathcal{R}_{G^{\prime}}^{0}$ is characterized by

$$
\begin{aligned}
R_{1}^{\prime} & \leq \min \left\{r_{1}-1, r_{2}-1\right\} \\
R_{1}^{\prime}+R_{2}^{\prime} & \leq r_{3}-1 \\
2 R_{1}^{\prime}+R_{2}^{\prime} & \leq r_{1}+r_{2}-3+\left|\mathcal{E}_{3}\right| .
\end{aligned}
$$

Since we have from (31) that

$$
r_{1}+r_{2}-3+\left|\mathcal{E}_{3}\right| \geq \min \left\{r_{1}-1, r_{2}-1\right\}+r_{3}-1,
$$

(36) is also not active in $\mathcal{R}_{G^{\prime}}^{0}$; i.e., $\mathcal{R}_{G^{\prime}}^{0}$ is characterized by

$$
\begin{aligned}
R_{1}^{\prime} & \leq \min \left\{r_{1}-1, r_{2}-1\right\} \\
R_{1}^{\prime}+R_{2}^{\prime} & \leq r_{3}-1
\end{aligned}
$$

This finally ensures that $\left(R_{1}-\min _{k} r_{k}^{e}, R_{2}\right) \in \mathcal{R}_{G^{\prime}}^{0}$ for all $\left(R_{1}=r+1, R_{2}\right) \in$ $\mathcal{R}_{G}^{0}$.

4. $\left|\mathcal{E}_{123}\right|=\left|\mathcal{E}_{12}\right|=\left|\mathcal{E}_{13}\right|=\left|\mathcal{E}_{23}\right|=0$ and $\left|\mathcal{E}_{1}\right| \&\left|\mathcal{E}_{2}\right| \&\left|\mathcal{E}_{3}\right|>0 . \mathcal{R}_{G^{\prime}}^{0}$ is characterized by

$$
\begin{aligned}
R_{1}^{\prime} & \leq \min \left\{r_{1}-1, r_{2}-1, r_{3}-1\right\} \\
R_{1}^{\prime}+R_{2}^{\prime} & \leq r_{3}-1 \\
2 R_{1}^{\prime}+R_{2}^{\prime} & \leq r_{1}-1+r_{2}-1+\left|\mathcal{E}_{3}\right|-1
\end{aligned}
$$


Furthermore, $\min _{k} r_{k}^{e}=1$. Since $\left|\mathcal{E}_{123}\right|=\left|\mathcal{E}_{12}\right|=\left|\mathcal{E}_{13}\right|=\left|\mathcal{E}_{23}\right|=0$, we have that $\min \left\{r_{1}, r_{2}, r_{3}\right\}-1+r_{3}-1 \leq r_{1}-1+r_{2}-1+\left|\mathcal{E}_{3}\right|-1$, and $\mathcal{R}_{G^{\prime}}^{0}$ is thus characterized by

$$
\begin{aligned}
R_{1}^{\prime} & \leq \min \left\{r_{1}-1, r_{2}-1, r_{3}-1\right\} \\
R_{1}^{\prime}+R_{2}^{\prime} & \leq r_{3}-1
\end{aligned}
$$

This proves that $\left(R_{1}-\min _{k} r_{k}^{e}, R_{2}\right) \in \mathcal{R}_{G^{\prime}}^{0}$ for all $\left(R_{1}=r+1, R_{2}\right) \in \mathcal{R}_{G}^{0}$.

5. $\left|\mathcal{E}_{123}\right|=\min \left\{\left|\mathcal{E}_{12}\right|,\left|\mathcal{E}_{13}\right|,\left|\mathcal{E}_{23}\right|\right\}=\min \left\{\left|\mathcal{E}_{i}\right|,\left|\mathcal{E}_{j, l}\right|\right\}=\min \left\{\left|\mathcal{E}_{1}\right|,\left|\mathcal{E}_{2}\right|,\left|\mathcal{E}_{3}\right|\right\}=$ $0 \forall\{i, j, l\}=\{1,2,3\}$, and $\left|\mathcal{E}_{i, j}\right| \&\left|\mathcal{E}_{i, l}\right|>0$ for some $\{i, j, l\}=\{1,2,3\}$.

- $i=3 . \mathcal{R}_{G^{\prime}}^{0}$ is characterized by

$$
\begin{aligned}
R_{1}^{\prime} & \leq \min \left\{r_{1}-1, r_{2}-1, r_{3}-1\right\} \\
R_{1}^{\prime}+R_{2}^{\prime} & \leq r_{3}-2 \\
2 R_{1}^{\prime}+R_{2}^{\prime} & \leq r_{1}-1+r_{2}-1+\left|\mathcal{E}_{3}\right| .
\end{aligned}
$$

Furthermore, $\min _{k} r_{k}^{e}=1$. Using the fact that $\left|\mathcal{E}_{123}\right|=\left|\mathcal{E}_{12}\right|=\left|\mathcal{E}_{1}\right|=$ $\left|\mathcal{E}_{2}\right|=0$, we show that $r_{1}+r_{2}-2+\left|\mathcal{E}_{3}\right|=r_{3}-2$ and thus $\mathcal{R}_{G^{\prime}}^{0}$ is actually characterized by

$$
\begin{aligned}
R_{1}^{\prime} & \leq \min \left\{r_{1}-1, r_{2}-1\right\} \\
2 R_{1}^{\prime}+R_{2}^{\prime} & \leq r_{1}-1+r_{2}-1+\left|\mathcal{E}_{3}\right| .
\end{aligned}
$$

Thus $\left(R_{1}-\min _{k} r_{k}^{e}, R_{2}\right) \in \mathcal{R}_{G^{\prime}}^{0}$ for all $\left(R_{1}=r+1, R_{2}\right) \in \mathcal{R}_{G}^{0}$.

- $i \neq 3$. $\mathcal{R}_{G^{\prime}}^{0}$ is characterized by

$$
\begin{aligned}
R_{1}^{\prime} & \leq \min \left\{r_{i}-2, r_{j}-1, r_{3}-1\right\} \\
R_{1}^{\prime}+R_{2}^{\prime} & \leq r_{3}-1 \\
2 R_{1}^{\prime}+R_{2}^{\prime} & \leq r_{1}+r_{2}-3+\left|\mathcal{E}_{3}\right| .
\end{aligned}
$$

Furthermore, $\min _{k} r_{k}^{e}=1$. By (37), we conclude that $\mathcal{R}_{G^{\prime}}^{0}$ is characterized by

$$
\begin{aligned}
R_{1}^{\prime} & \leq \min \left\{r_{j}-1, r_{i}-2\right\} \\
R_{1}^{\prime}+R_{2}^{\prime} & \leq r_{3}-1 .
\end{aligned}
$$

Furthermore, $r_{j}=\left|\mathcal{E}_{12}\right|<\left|\mathcal{E}_{i 3}\right|+\left|\mathcal{E}_{i}\right|+\left|\mathcal{E}_{12}\right|=r_{i}$ and so $r_{j}-1 \leq r_{i}-2$, ensuring $\left(R_{1}-\min _{k} r_{k}^{e}, R_{2}\right) \in \mathcal{R}_{G^{\prime}}^{0}$ for all $\left(R_{1}=r+1, R_{2}\right) \in \mathcal{R}_{G}^{0}$. 


\subsection{Algorithm optimality - erasures}

In this section we assume the erasure probability $\alpha>0$ for all the channels of the combination network independently and that messages $W_{1}$ and $W_{2}$ of rates $\left(R_{1}, R_{2}\right) \in \mathcal{R}_{G}^{\alpha}$ are to be communicated over the combination network. We use a random code of rate $(1-\alpha)$ and encode the $n R_{1}$ symbols of $W_{1}$ to $\frac{n R_{1}}{1-\alpha}$ symbols and similarly $W_{2}$ symbols to $\frac{n R_{2}}{1-\alpha}$ symbols of $W_{2}$. Linear combinations of encoded $W_{1}$ and also of encoded $W_{2}$ symbols are now of a rate smaller than $1-\alpha$ and can thus be communicated with arbitrary small error probability to intermediate nodes. We can thus as before apply the Algorithm 2 algorithm with a rate pair $\left(\frac{n R_{1}}{1-\alpha}, \frac{n R_{2}}{1-\alpha}\right)$. We just have to show that

$$
\operatorname{Pr}\left\{W_{1} \neq \hat{W}_{1}\right\} \stackrel{n \rightarrow \infty}{\rightarrow} 0
$$

Since the receivers are provided with random linear combinations of encoded message $W_{1}$ and random linear combinations of encoded message $W_{2}$, (55) holds if the following two conditions are satisfied with high probability:

- The number of non-erased $W_{1}$ carrying signals received at each receiver is greater than or equal to $n R_{1}$ with high probability, and

- The number of non-erased $W_{2}$ carrying signals received at receiver 3 is greater than or equal to $n R_{2}$ with high probability.

Consider the received vector $\bar{Y}_{i}^{n}$ at receiver $i$. By the algorithm analysis in Section 4.1, we know that each receiver $i$ is connected to at least $\frac{n R_{1}}{1-\alpha}$ edges which carry linear combinations of the randomly encoded $W_{1}$ (with high probability). Pick the set (of cardinality $\frac{n R_{1}}{1-\alpha}$ ) of those edges carrying the aforementioned $\frac{n R_{1}}{1-\alpha}$ linear combinations. By some abuse of notation, call them $Y_{1}, \cdots, Y_{\frac{n R_{1}}{1-\alpha}}$. Assign to each $Y_{k}$ a random variable $Z_{k}$ defined as

$$
Z_{k}=\left\{\begin{array}{ll}
0 & \text { if } Y_{k} \text { is erased } \\
1 & \text { otherwise }
\end{array} .\right.
$$

Since $\operatorname{Pr}\left\{\left|\sum_{k} Z_{k}-\frac{n R_{1}}{1-\alpha} \times(1-\alpha)\right| \geq \epsilon\right\} \rightarrow 0$ when $n \rightarrow \infty$, the number of non-erased $W_{1}$ carrying signals received at each receiver is greater than or equal to $n R_{1}$ with high probability. Similarly for $W_{2}$. This concludes the achievability of the rate pair $\left(R_{1}, R_{2}\right) \in \mathcal{R}_{G}^{\alpha}$. 


\section{Algorithm evaluation}

In this section we compare the encoding scheme given by Algorithm 2 described in previous sections, with a naive network coding-based scheme, which we denote by $N C$ naive, from the point of view of the total number of time slots needed to deliver a certain rate with each of the schemes. We assume throughout this section that the erasure probability $\alpha=0$.

For the NC naive scheme, the source has only information about the min-cut of each receiver, and it does not know which edge is visible to what receiver. The server starts by sending linear combinations corresponding to message $W_{1}$ until all receivers decode it, after which it continues with sending linear combinations of message $W_{2}$ until receiver 3 decodes it.

Consider we use the network during $T$ time slots. For any rate pair $\left(R_{1}, R_{2}\right) \in$ $\mathcal{R}_{G}^{0}$, the Algorithm 2 scheme delivers a total rate of $T\left(R_{1}+R_{2}\right)$, as in each time slot it is able to assign the resources such that to achieve the desired rate pair. Thus, with Algorithm 2 the server is sending at rate $R_{1}+R_{2}$ during each time slot.

For the purpose of our analysis, let us consider that with $N C$ naive, the server is sending at the same rate during each time slot as with Algorithm 2. That means a destination $i$ will receive a useful rate of $\left(R_{1}+R_{2}\right) \frac{r_{i}}{r_{123}}$ on average during each time slot. Consequently, in order to deliver a total rate of $T R_{1}$ of $W_{1}$ to all receivers and a total rate of $T R_{2}$ of $W_{2}$ to destination 3, the server needs to use the network during $T_{n}=T_{1}+T_{2}$ time slots, where:

$$
T_{1}=\frac{T R_{1}}{\left(R_{1}+R_{2}\right) \frac{\min \left\{r_{i}\right\}}{r_{123}}},
$$

and

$$
T_{2}=\frac{T R_{2}}{\left(R_{1}+R_{2}\right) \frac{r_{3}}{r_{123}}} .
$$

Next we define the function

$$
f\left(R_{1}, R_{2}\right)=\frac{T_{n}}{T}=\frac{r_{123}\left(\frac{R_{1}}{\min \left\{r_{i}\right\}}+\frac{R_{2}}{r_{3}}\right)}{R_{1}+R_{2}},
$$

where $R_{1}$ and $R_{2}$ cannot be simultaneously 0 .

Note that if $f\left(R_{1}, R_{2}\right)$ takes higher values, this means the time needed to deliver a desired rate is significantly shorter for the scheme proposed by Algorithm 2 


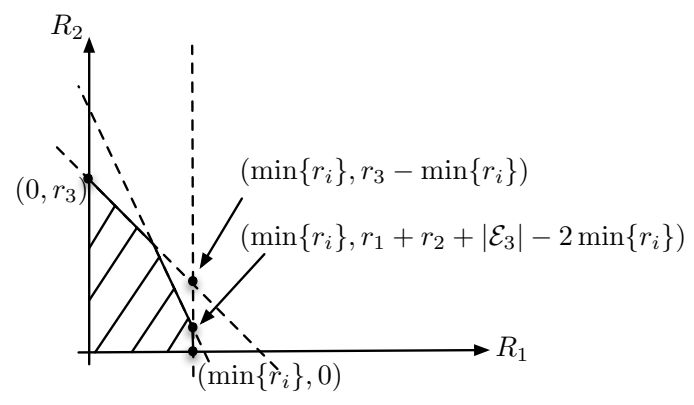

Figure 3: The critical points

as compared to the naive approach. Thus we are interested to find out for which rate pairs $\left(R_{1}, R_{2}\right) \in \mathcal{R}_{G}^{0}$ does the Algorithm 2 scheme yield most of the benefits and in what type of topologies we are most likely to get benefits. To this end, we solve the following optimization problem:

$$
\begin{aligned}
\max f\left(R_{1}, R_{2}\right) & \\
R_{1} & \leq \min \left\{r_{i}\right\} \\
R_{1}+R_{2} & \leq r_{3} \\
2 R_{1}+R_{2} & \leq r_{1}+r_{2}+\left|\mathcal{E}_{3}\right| .
\end{aligned}
$$

We begin our analysis with two observations. First, note that for those topologies where the third receiver is the bottleneck, in the sense that he sees the least number of edges, i.e. $r_{3}=\min \left\{r_{i}\right\}$, our function becomes constant for any rate pair in the rate region: $f\left(R_{1}, R_{2}\right)=\frac{r_{123}}{r_{3}}$. Second, for those topologies where all the edges are visible to all the receivers, i.e. $r_{i}=r_{123}$ for $i=1,2,3$, our function $f\left(R_{1}, R_{2}\right)=1$, which means that Algorithm 2 does not yield any benefit over the naive approach. Intuitively, even if with $N C$ naive the server selects the outgoing edges at random, every packet sent on any edge is received by everyone, since all the destinations have access to all the edges.

In the following we solve the optimization problem for the case when $r_{3}>$ $\min \left\{r_{i}\right\}$ for $i \in\{1,2,3\}$ and discuss the case of the inequality (6) being active separately.

\section{Inequality (6) is not active}

We find the following critical points: 


$$
\begin{aligned}
& \left(R_{1}^{1}, R_{2}^{1}\right)=\left(\min \left\{r_{i}\right\}, 0\right) \\
& \left(R_{1}^{2}, R_{2}^{2}\right)=\left(\min \left\{r_{i}\right\}, r_{3}-\min \left\{r_{i}\right\}\right) \\
& \left(R_{1}^{3}, R_{2}^{3}\right)=\left(0, r_{3}\right)
\end{aligned}
$$

for which our function takes these values:

$$
\begin{aligned}
f\left(R_{1}^{1}, R_{2}^{1}\right) & =\frac{r_{123}}{\min \left\{r_{i}\right\}}, \\
f\left(R_{1}^{2}, R_{2}^{2}\right) & =\frac{r_{123}}{r_{3}}\left(2-\frac{\min \left\{r_{i}\right\}}{r_{3}}\right), \\
f\left(R_{1}^{3}, R_{2}^{3}\right) & =\frac{r_{123}}{r_{3}} .
\end{aligned}
$$

Further note that $f\left(R_{1}^{1}, R_{2}^{1}\right)>f\left(R_{1}^{2}, R_{2}^{2}\right)>f\left(R_{1}^{3}, R_{2}^{3}\right)$.

\section{Inequality (6) is active}

In this case, in addition to $\left(R_{1}^{1}, R_{2}^{1}\right)$ and $\left(R_{1}^{3}, R_{2}^{3}\right)$ we also find:

$$
\left(R_{1}^{4}, R_{2}^{4}\right)=\left(\min \left\{r_{i}\right\}, r_{1}+r_{2}+\left|\mathcal{E}_{3}\right|-2 \min \left\{r_{i}\right\}\right),
$$

with

$$
f\left(R_{1}^{4}, R_{2}^{4}\right)=\frac{r_{123}}{r_{3}}\left(1+\frac{r_{3}-\min \left\{r_{i}\right\}}{r_{1}+r_{2}+\left|\mathcal{E}_{3}\right|-\min \left\{r_{i}\right\}}\right) .
$$

Finally note that $f\left(R_{1}^{1}, R_{2}^{1}\right)>f\left(R_{1}^{4}, R_{2}^{4}\right)>f\left(R_{1}^{3}, R_{2}^{3}\right)$.

\section{Conclusions and future work}

In this paper we studied the degraded two message set problem, over a combination network $G$ and in the presence of erasures of rate $\alpha$. We gave a characterization of the rate region, $\mathcal{R}_{G}^{\alpha}$, and introduced an algorithm that achieves it by using topological information. Further we compared our algorithm to a naive approach that selects the resources at random, and discussed the benefits that should be expected in different classes of networks.

As future work, we consider extending the algorithm to the case of multicasting to a larger set of receivers and carry on a practical evaluation of the proposed algorithm. 


\section{References}

[1] C. Fragouli and E. Soljanin, "Network Coding Fundamentals", Monograph in Series, Foundations and Trends in Networking, 2007.

[2] T M. Cover. Broadcast channels. IEEE Transactions on Information Theory, 18:2-14, January 1972.

[3] P. Bergmans. A simple converse for broadcast channels with additive white Gaussian noise. IEEE Transactions on Information Theory, 20:279-280, March 1974.

[4] R G. Gallager. Capacity and coding for degraded broadcast channels. Problemy Peredachi Informatsii, 10(3):3-14, 1974.

[5] J. Korner and K. Marton. General broadcast channels with degraded message sets. IEEE Trans. IT, 23(1):60-64, January 1977.

[6] S. Mohajer, S N. Diggavi and D. Tse, "Approximate Capacity of a Class of Gaussian Relay-Interference Networks," IEEE International Symposium on Information Theory, Seoul, Korea, June 2009.

[7] C. Nair and A. El Gamal, "The Capacity Region of a Class of 3-Receiver Broadcast Channels with Degraded Message Sets", Proceedings of the International Symposium on Information Theory, pp. 1706-1710, Toronto, June 2008.

[8] S. Saeedi, S. Diggavi, C. Fragouli, and V. Prabhakaran, "On degraded two message set broadcasting", IEEE ITW 2009.

[9] S. Gheorghiu, L. Lima, A. Lopez Toledo, J. Barros, M. Medard, "On the Performance of Network Coding in Multi-Resolution Wireless Video Streaming“, 2010 IEEE International Symposium on Network Coding 